\title{
THE NEHARI MANIFOLD APPROACH FOR SINGULAR EQUATIONS INVOLVING THE $p(x)$-LAPLACE OPERATOR
}

\author{
DUŠAN D. REPOVŠ \\ Faculty of Education and Faculty of Mathematics and Physics, University of \\ Ljubljana \& Institute of Mathematics, Physics and Mechanics, 1000 Ljubljana, \\ Slovenia \\ Email: dusan.repovs@guest.arnes.si \\ KAMEL SAOUdi \\ College of Sciences at Dammam, University of Dammam \\ 31441 Dammam, Kingdom of Saudi Arabia \\ Email:kmsaoudi@iau.edu.sa
}

\begin{abstract}
We study the following singular problem involving the $\mathrm{p}(x)$-Laplace operator $\Delta_{p(x)} u=\operatorname{div}\left(|\nabla u|^{p(x)-2} \nabla u\right)$, where $p(x)$ is a nonconstant continuous function,

$$
\left(\mathrm{P}_{\lambda}\right)\left\{\begin{aligned}
-\Delta_{p(x)} u & =a(x)|u|^{q(x)-2} u(x)+\frac{\lambda b(x)}{u^{\delta(x)}} \quad \text { in } \Omega, \\
u & >0 \quad \text { in } \Omega, \\
u & =0 \quad \text { on } \partial \Omega .
\end{aligned}\right.
$$

Here, $\Omega$ is a bounded domain in $\mathbb{R}^{N \geq 2}$ with $C^{2}$-boundary, $\lambda$ is a positive parameter, $a(x), b(x) \in C(\bar{\Omega})$ are positive weight functions with compact support in $\Omega$, and $\delta(x), p(x), q(x) \in C(\bar{\Omega})$ satisfy certain hypotheses $\left(A_{0}\right)$ and $\left(A_{1}\right)$. We apply the Nehari manifold approach and some new techniques to establish the multiplicity of positive solutions for problem $\left(\mathrm{P}_{\lambda}\right)$.

Keywords: Nehari manifold, generalized Lebesgue-Sobolev space, topological method, singular equation, $\mathrm{p}(x)$-Laplace operator, multiplicity.
\end{abstract}

\section{INTRODUCTION}

The aim of this paper is to study the following inhomogeneous equation

$$
\left(\mathrm{P}_{\lambda}\right)\left\{\begin{aligned}
-\Delta_{p(x)} u & =a(x)|u|^{q(x)-2} u(x)+\frac{\lambda b(x)}{u^{\delta(x)}} \quad \text { in } \Omega, \\
u & >0 \quad \text { in } \Omega, \\
u & =0 \quad \text { on } \partial \Omega .
\end{aligned}\right.
$$

Here, operator $\Delta_{p(x)} u:=\operatorname{div}\left(|\nabla u|^{p(x)-2} \nabla u\right)$ is the $p(x)$-Laplacian, $p(x)$ is a nonconstant continuous function, $\Omega$ is a bounded domain in $\mathbb{R}^{N}(N \geq 2)$ with $C^{2}$ boundary, $\lambda$ is a positive parameter, $a(x), b(x) \in C(\bar{\Omega})$ are positive weight functions with compact support in $\Omega$, and $\delta(x), p(x), q(x) \in C(\bar{\Omega})$ satisfy the following conditions

Math. Subj. Classif. (2010): 35J20, 35J60, 35J70, 47J10. 
$\left(A_{0}\right) 0<1-\delta(x)<p(x)<q(x)<p^{*}(x)$

$\left(A_{1}\right) 0<1-\delta^{-} \leq 1-\delta^{+}<p^{-} \leq p^{+}<q^{-} \leq q^{+}$.

Here, $p^{*}(x):=N p(x) /(N-p(x)), \delta^{+}:=\operatorname{ess} \sup \delta(x), \delta^{-}:=\operatorname{essinf} \delta(x)$, and analogous definitions hold for $p^{-}, p^{+}, q^{-}$, and $q^{+}$.

Partial differential equations with variable exponents are a very interesting and active topics. The motivation for this type of problems was stimulated by their various applications in physics - for more details see ACERBi-Mingione [1], DienING [4], and in particular the book RĂDULESCU-REPOVŠ [15]) and the references therein.

Before stating our main result, we review the key literature concerning singular partial differential equations with variable exponents. ZHANG [25] proved the existence of solutions for the purely singular problem. Using variational methods, SAOUDI [17] proved the existence for a superlinear singular equation with variable exponent. FAN [6] investigated the multiplicity of solutions using topological methods. In SAoudi-Ghanmi [20] and SAoudi ET AL. [23] variational methods were used to establish the multiplicity of solutions for a singular problems with Dirichlet and Neumann conditions, respectively (see also SAOudi-GHANmi [21]).

The case when $p$ is constant in problem $\left(\mathrm{P}_{\lambda}\right)$, has recieved more attention and has been approached by differents techniques. For a more general presentation we refer to Coclite-Palmieri [2], Crandall et al. [3], Ghergu-Rădulescu [9], Giacomoni-Saoudi [10], Giacomoni et al. [11], Saoudi [18], and SaoudiKRATOU [22] and the references therein.

Some interesting papers on the applications of the Nehari manifold method in variable exponent problem have recently been published, see e.g. MASHIYEV ET al. [13], Saiedinezhad-Ghaemi [16], and Saoudi [19]. In the present paper, we generalize the results of GIACOMONI ET AL. [11] and SAOUd [18] to the problem with variable exponent, by using topological methods. Here is the main result of this paper.

Theorem 1.1. Suppose that condition $\left(A_{0}\right)$ and $\left(A_{1}\right)$ are fulfilled. Then there exists $\lambda_{0}>0$ such that for every $\lambda \in\left(0, \lambda_{0}\right)$, problem $\left(\mathrm{P}_{\lambda}\right)$ has at least two positive solutions.

This paper is organized as follows. In Section 2, we briefly review the properties of generalized Lebesgue-Sobolev spaces. In Section 3, we prove the necessary lemmas. In Section 4, we prove the existence of a minimum for the functional energy $E_{\lambda}$ in $\mathcal{N}_{\lambda}^{+}$. In Section 5, we prove the existence of a minimum for the functional energy $E_{\lambda}$ in $\mathcal{N}_{\lambda}^{-}$. Finally, In Section 6 , we present the proof of our main result.

\section{Generalized Lebesgue-Sobolev Spaces}

In this section, we recall definitions of functional spaces with variable exponents and properties of the $p(x)$-Laplacian operator which will be used later (for more on this topics see RăDULESCU-REPOVŠ [15], and for other additional information see PAPAgeorgiou ET AL. [14]). Let

$$
L^{p(\cdot)}(\Omega)=\left\{u \in S(\Omega): \int_{\Omega}|u(x)|^{p(x)} d x<\infty\right\},
$$


with the norm

$$
|u|_{p(\cdot)}=|u|_{L^{p(\cdot)}(\Omega)}=\inf \left\{\lambda>0: \int_{\Omega}\left|\frac{u(x)}{\lambda}\right|^{p(x)} d x \leq 1\right\} .
$$

Then $\left(L^{p(\cdot)}(\Omega),|\cdot|_{p(\cdot)}\right)$ is a reflexive, uniform convex Banach, separable space - for details see FAN-ZhaO [8, Theorems 1.6, 1.10, 1.14] and RĂDUlESCU-REPOVŠ [15].

The variable exponent Sobolev space

$$
W^{1, p(\cdot)}(\Omega)=\left\{u \in L^{p(\cdot)}(\Omega):|\nabla u| \in L^{p(\cdot)}(\Omega)\right\},
$$

can be equipped with the norm

$$
\|u\|=|u|_{p(\cdot)}+|\nabla u|_{p(\cdot)}, \quad \text { for all } u \in W^{1, p(\cdot)}(\Omega) .
$$

Note that $W_{0}^{1, p(\cdot)}(\Omega)$ is the closure of $C_{0}^{\infty}(\Omega)$ in $W^{1, p(\cdot)}(\Omega)$.

We denote by $L^{q(x)}(\Omega)$ the conjugate space of $L^{p(x)}(\Omega)$, where $\frac{1}{q(x)}+\frac{1}{p(x)}=1$. For $u \in L^{p(x)}(\Omega)$ and $v \in L^{q(x)}(\Omega)$, the Hölder type inequality

$$
\left|\int_{\Omega} u(x) v(x) d x\right| \leq\left(\frac{1}{p^{-}}+\frac{1}{q^{-}}\right)|u|_{p(x)}|v|_{q(x)},
$$

holds. Recall the following result.

Lemma 2.1. Consider the mapping $\rho_{p(x)}: L^{p(x)}(\Omega) \rightarrow \mathbb{R}$ defined by

$$
\rho_{p(x)}(u)=\int_{\Omega}|u|^{p(x)} d x,
$$

where $\left(u_{n}\right), u \in L^{p(x)}(\Omega)$, and $p^{+}<\infty$. Then the following relations hold

$$
\begin{gathered}
\|u\|_{L^{p(x)}}>1 \Rightarrow\|u\|_{L^{p(x)}}^{p^{-}} \leq \rho_{p(x)}(u) \leq\|u\|_{L^{p(x)}}^{p^{+}}, \\
\|u\|_{L^{p(x)}}<1 \Rightarrow\|u\|_{L^{p(x)}}^{p^{+}} \leq \rho_{p(x)}(u) \leq\|u\|_{L^{p(x)}}^{p^{-}}, \\
\left\|u_{n}-u\right\|_{L^{p(x)}} \rightarrow 0 \text { if and only if } \rho_{p(x)}\left(u_{n}-u\right) \rightarrow 0 .
\end{gathered}
$$

We state the Sobolev embedding theorem.

Theorem 2.1 ( See FAN ET AL. [7] and KovăČIK-RĂKOSNIK [12]). Let $p \in C(\bar{\Omega})$ with $p(x)>1$ for each $x \in \bar{\Omega}$ where $\Omega \subset \mathbb{R}^{N}$ is an open bounded domain with Lipschitz boundary and suppose that $p(x) \leq r(x) \leq p^{*}(x)$ and $r \in C(\bar{\Omega})$, for all $x \in \bar{\Omega}$. Then the embedding $W^{1, p(x)}(\Omega) \hookrightarrow L^{r(x)}(\Omega)$ is continuous. Also, if $r(x)<$ $p^{*}(x)$ almost everywhere in $\bar{\Omega}$, then this embedding is compact.

Let $\rho(x, s)$ be a Carathéodory function satisfying the following condition

$$
|\rho(x, s)| \leq A \text { for a.e. } x \in \Omega \text { and all } s \in\left[-s_{0}, s_{0}\right],
$$

where $s_{0}>0$ and $A$ is a constant. Recall the following comparison principle.

Lemma 2.2 (ZhANG [24, Lemma 2.3]). Let $\rho(x, t)$ be a function satisfying (2.5) and increasing in $t$. Let $u, v \in W^{1, p(\cdot)}(\Omega)$ satisfy

$$
-\Delta_{p(x)} u+\rho(x, u) \leq-\Delta_{p(x)} v+\rho(x, v), \quad \text { for all } x \in \Omega .
$$

and assume that $u \leq v$ on $\partial \Omega$. Then $u \leq v$ in $\Omega$.

Next, we recall the following strong maximum principle. 
Theorem 2.2 ( SaOudi-Ghanmi [20, Theorem 3.2]). Suppose that for some $0<$ $\alpha<1, u, v \in C^{1, \alpha}(\bar{\Omega})$ we have $0 \supsetneqq u, 0 \supsetneqq v$, and

$$
-\Delta_{p(x)} u-\frac{\lambda}{u^{\delta(x)}}=h(x) \geq g(x)=-\Delta_{p(x)} v-\frac{\lambda}{v^{\delta(x)}},
$$

with $u=v=0$ on $\partial \Omega$, where $g, h \in L^{\infty}(\Omega)$ are such that $0 \leq g<h$ pointwise everywhere in $\Omega$. Assume that

$$
\frac{\partial u}{\partial \mathbf{n}}>0 \quad \frac{\partial v}{\partial \mathbf{n}}>0 \text { on } \partial \Omega,
$$

where $\mathbf{n}$ is the inward unit normal on $\partial \Omega$. Then the following strong comparison principle holds:

$u>v$ in $\Omega, \quad$ and there is a positive $\epsilon$ such that $\frac{\partial(u-v)}{\partial \mathbf{n}} \geq \epsilon$ on $\partial \Omega$.

We shall now prove the following result.

Theorem 2.3. Suppose that the domain $\Omega$ has the cone property and consider $p \in C(\bar{\Omega})$. Assume that $b \in L^{\alpha(x)}, b(x)>0$ for $x \in \Omega, \alpha \in C(\bar{\Omega})$ and $\alpha^{-}>$ $1, \alpha_{0}^{-} \leq \alpha_{0}(x) \leq \alpha_{0}^{+}\left(\frac{1}{\alpha(x)}+\frac{1}{\alpha_{0}(x)}=1\right), \delta \in C(\bar{\Omega})$, and

$$
0<1-\delta(x)<\frac{\alpha(x)-1}{\alpha(x)} p^{*}(x), \text { for all } x \in \bar{\Omega} .
$$

Then the embedding $W^{1, p(x)}(\Omega) \hookrightarrow L_{b(x)}^{1-\delta(x)}(\Omega)$ is compact. Moreover, there is a constant $c_{2}>0$ such that the following inequality holds

$$
\int_{\Omega} b(x)|u|^{1-\delta(x)} d x \leq c_{2}\left(\|u\|^{1-\delta^{-}}+\|u\|^{1-\delta^{+}}\right) .
$$

Proof. The proof of the first assertion is adopted from FAN [6]. Let $u \in W^{1, p(x)}(\Omega)$ and let

$$
r(x)=\frac{\alpha(x)}{\alpha(x)-1}(1-\delta(x))=\alpha_{0}(x)(1-\delta(x)) .
$$

Hence, (2.9) implies that $r(x)<p^{*}(x)$. Therefore, using Theorem (2.1), we obtain $W^{1, p(x)}(\Omega) \hookrightarrow L^{r(x)}(\Omega)$. So, for $u \in W^{1, p(x)}(\Omega)$, we get $|u|^{1-\delta(x)} \in L^{\alpha_{0}(x)}(\Omega)$. By $(2.1)$,

$$
\int_{\Omega} b(x)|u|^{1-\delta(x)} d x \leq\left. c_{1}|b|_{\alpha(x)}|| u\right|^{1-\delta(x)} \mid<\infty .
$$

This means that $W^{1, p(x)}(\Omega) \subset L^{1-\delta(x)}(\Omega)$.

On the other hand, if $u_{n} \rightarrow 0$ weakly in $W^{1, p(x)}(\Omega)$, then we have that $u_{n} \rightarrow$ 0 strongly in $L^{r(x)}(\Omega)$. Therefore,

$$
\int_{\Omega} b(x)\left|u_{n}\right|^{1-\delta(x)} d x \leq\left. c_{1}|b|_{\alpha(x)}|| u_{n}\right|^{1-\delta(x)} \mid \rightarrow 0,
$$

hence $\left|u_{n}\right|_{1-\delta(x), b(x)} \rightarrow 0$ and we can conclude that

$$
W^{1, p(x)}(\Omega) \hookrightarrow L_{b(x)}^{1-\delta(x)}(\Omega) .
$$

Next, we shall prove inequality (2.10). First, we have from above

$$
\int_{\Omega} b(x)|u|^{1-\delta(x)} d x \leq\left. c_{1}|b|_{\alpha(x)}|| u\right|^{1-\delta(x)} \mid<\infty .
$$


Since $1-\delta^{-} \leq 1-\delta(x) \leq 1-\delta^{+}$and $|u|^{1-\delta(x)} \leq|u|^{1-\delta^{-}}+|u|^{1-\delta^{+}}$, we obtain

$$
\int_{\Omega} b(x)|u|^{1-\delta(x)} d x \leq \int_{\Omega} b(x)|u|^{1-\delta^{-}} d x+\int_{\Omega} b(x)|u|^{1-\delta^{+}} d x .
$$

On the other hand, using (2.1), (2.2), (2.3), (2.4), and condition $p(x)<(1-$ $\left.\delta^{-}\right) \alpha_{0}(x) \leq\left(1-\delta^{+}\right) \alpha_{0}(x)<p^{*}(x)$, we get

$$
\int_{\Omega} b(x)|u|^{1-\delta^{-}} d x \leq\left.\left. c_{2}|b|_{\alpha(x)}|| u\right|^{1-\delta(x)}\right|_{\alpha_{0}(x)}=c_{2}|b|_{\alpha(x)}|u|_{\left(1-\delta^{-}\right) \alpha_{0}(x)}^{1-\delta^{-}} \leq c_{3}|| u||^{1-\delta^{-}} .
$$

In the same way, one gets

$$
\int_{\Omega} b(x)|u|^{1-\delta^{+}} d x \leq c_{4}\|u\|^{1-\delta^{+}} .
$$

Hence, using (2.11) and (2.12), we have

$$
\int_{\Omega} b(x)|u|^{1-\delta(x)} d x \leq c_{5}\left(\|u\|^{1-\delta^{-}}+\|u\|^{1-\delta^{+}}\right) .
$$

which completes the proof of Theorem 2.3.

Theorem 2.4. Let $p \in C(\bar{\Omega})$, suppose the boundary of domain $\Omega$ has the cone property and let $u \in W^{1, p(x)}(\Omega)$. Then there exist nonnegative constants $c_{6}, c_{7}, c_{8}, c_{9}>$ 0 such that the following inequalities hold

$$
\begin{gathered}
\int_{\Omega} a(x)|u|^{q(x)} d x \leq \begin{cases}c_{6}\|u\|^{q^{+}} & \text {if }\|u\|>1, \\
c_{7}\|u\|^{q^{-}} & \text {if }\|u\|<1 .\end{cases} \\
\int_{\Omega} b(x)|u|^{1-\delta(x)} d x \leq \begin{cases}c_{8}\|u\|^{1-\delta^{-}} & \text {if }\|u\|>1, \\
c_{9}\|u\|^{1-\delta^{+}} & \text {if }\|u\|<1 .\end{cases}
\end{gathered}
$$

Proof. Theorem 2.4 follows immediately by MASHIYEV ET AL. [13, Theorem 2.3] and Theorem 2.3.

\section{SOME NECESSARY LEMMAS}

Let us define the functional $E_{\lambda}: W_{0}^{1, p(x)}(\Omega) \rightarrow \mathbb{R}$ by

$$
E_{\lambda}(u) \stackrel{\text { def }}{=} \int_{\Omega} \frac{|\nabla u|^{p(x)}}{p(x)} d x-\int_{\Omega} \frac{a(x)|u|^{q(x)}}{q(x)} d x-\lambda \int_{\Omega} \frac{b(x)\left(u^{+}\right)^{1-\delta(x)}}{1-\delta(x)} d x(3.1)
$$

Definition 3.1. We say that $u \in W_{0}^{1, p(x)}(\Omega)$ is a generalized solution of the equation

$$
-\Delta_{p(x)} u=a(x)|u|^{q(x)-2} u(x)+\frac{\lambda b(x)}{u^{\delta(x)}}
$$

if for all $\varphi \in C_{0}^{\infty}(\Omega)$ and $\underset{K}{\operatorname{ess} \inf } u>0$ for every compact set $K \subset \Omega$,

$$
\int_{\Omega}|\nabla u|^{p(x)-2} \nabla u \nabla \varphi d x=\int_{\Omega} a(x)|u|^{q(x)-1} \varphi d x+\lambda \int_{\Omega} b(x) u^{-\delta(x)} \varphi d x
$$

for all $\varphi \in C_{0}^{\infty}(\Omega)$. 
Obviously, every weak solution of problem $\left(P_{\lambda}\right)$ is also a generalized solution of equation (3.2).

In many problems, such as $\left(P_{\lambda}\right), E_{\lambda}$ is not bounded below on $W_{0}^{1, p(x)}(\Omega)$, but it is bounded below on the corresponding Nehari manifold which is defined by

$$
\mathcal{N}_{\lambda}:=\left\{u \in W_{0}^{1, p(x)}(\Omega) \backslash\{0\}:\left\langle E_{\lambda}^{\prime}(u), u\right\rangle=0\right\} .
$$

Then $u \in \mathcal{N}_{\lambda}$ if and only if

$$
\int_{\Omega} \frac{|\nabla u|^{p(x)}}{p(x)} d x-\int_{\Omega} \frac{a(x)|u|^{q(x)}}{q(x)} d x-\lambda \int_{\Omega} \frac{b(x)|u|^{1-\delta(x)}}{1-\delta(x)} d x=0 .
$$

We note that $\mathcal{N}_{\lambda}$ contains every solution of problem $\left(P_{\lambda}\right)$.

It is well-known that the Nehari manifold is closely related to the behavior of the functions $\Phi_{u}:[0, \infty) \rightarrow \mathbb{R}$ defined as $\Phi_{u}(t)=E_{\lambda}(t u)$. Such maps are called fiber maps and were introduced by DrabeK-Pohozaev [5]. For $u \in W_{0}^{1, p(x)}(\Omega) \backslash\{0\}$, we define

$$
\begin{aligned}
\Phi_{u}(t) & =\int_{\Omega} \frac{t^{p(x)}|\nabla u|^{p(x)}}{p(x)} \mathrm{d} x-\int_{\Omega} \frac{a(x) t^{q(x)}}{q(x)}|u|^{q(x)} d x-\lambda \int_{\Omega} \frac{b(x) t^{1-\delta(x)}|u|^{1-\delta(x)}}{1-\delta(x)} d x, \\
\Phi_{u}^{\prime}(t) & =\int_{\Omega} t^{p(x)-1}|\nabla u|^{p(x)} \mathrm{d} x-\int_{\Omega} a(x) t^{q(x)-1}|u|^{q(x)} d x-\lambda \int_{\Omega} b(x) t^{-\delta(x)}|u|^{1-\delta(x)} d x, \\
\Phi_{u}^{\prime \prime}(t) & =\int_{\Omega}(p(x)-1) t^{p(x)-2}|\nabla u|^{p(x)} \mathrm{d} x-\int_{\Omega} a(x)(q(x)-1) t^{q(x)-2}|u|^{q(x)} d x \\
& +\lambda \int_{\Omega} b(x) \delta(x) t^{-\delta(x)-1}|u|^{1-\delta(x)} d x .
\end{aligned}
$$

It is easy to see that $t u \in \mathcal{N}_{\lambda}$ if and only if $\Phi_{u}^{\prime}(t)=0$ and in particular, $u \in \mathcal{N}_{\lambda}$ if and only if $\Phi_{u}^{\prime}(1)=0$. Thus it is natural to split $\mathcal{N}_{\lambda}$ into three parts corresponding to local minima, local maxima and points of inflection defined as follows:

$$
\begin{aligned}
& \mathcal{N}_{\lambda}^{+}:=\left\{u \in \mathcal{N}_{\lambda}: \Phi_{u}^{\prime \prime}(1)>0\right\}=\left\{t u \in W_{0}^{1, p(x)}(\Omega) \backslash\{0\}: \Phi_{u}^{\prime}(t)=0, \Phi_{u}^{\prime \prime}(t)>0\right\}, \\
& \mathcal{N}_{\lambda}^{-}:=\left\{u \in \mathcal{N}_{\lambda}: \Phi_{u}^{\prime \prime}(1)<0\right\}=\left\{t u \in W_{0}^{1, p(x)}(\Omega) \backslash\{0\}: \Phi_{u}^{\prime}(t)=0, \Phi_{u}^{\prime \prime}(t)<0\right\}, \\
& \mathcal{N}_{\lambda}^{0}:=\left\{u \in \mathcal{N}_{\lambda}: \Phi_{u}^{\prime \prime}(1)=0\right\}=\left\{t u \in W_{0}^{1, p(x)}(\Omega) \backslash\{0\}: \Phi_{u}^{\prime}(t)=0, \Phi_{u}^{\prime \prime}(t)=0\right\} .
\end{aligned}
$$

Our first result is the following.

Lemma 3.1. $E_{\lambda}$ is coercive and bounded below on $\mathcal{N}_{\lambda}$.

Proof. Let $u \in \mathcal{N}_{\lambda}$ and $\|u\|>1$. Then, using (2.2)-(2.4) and the embeddings from Theorem 2.1, we estimate $E_{\lambda}(u)$ as follows:

$$
\begin{aligned}
E_{\lambda}(u) & =\int_{\Omega} \frac{|\nabla u|^{p(x)}}{p(x)} d x-\int_{\Omega} \frac{a(x)|u|^{q(x)}}{q(x)} d x-\lambda \int_{\Omega} \frac{b(x)|u|^{1-\delta(x)}}{1-\delta(x)} d x \\
& \geq\left(\frac{1}{p^{+}}-\frac{1}{q^{-}}\right) \int_{\Omega}|\nabla u|^{p(x)} d x-\lambda\left(\frac{1}{1-\delta^{+}}-\frac{1}{q^{-}}\right) \int_{\Omega} b(x)|u|^{1-\delta(x)} d x \\
& \geq\left(\frac{1}{p^{+}}-\frac{1}{q^{-}}\right)\|u\|^{p^{-}}-\lambda c_{8}\left(\frac{1}{1-\delta^{+}}-\frac{1}{q^{-}}\right)\|u\|^{1-\delta^{+}} .
\end{aligned}
$$

Note that since $0<\delta^{+}<1$ and $1-\delta^{+}<p^{-}$, it follows that $E_{\lambda}(u) \rightarrow \infty$ as $\|u\| \rightarrow \infty$. Therefore $E_{\lambda}$ is coercive and bounded below. 
Lemma 3.2. Let $u$ be a local minimizer for $E_{\lambda}$ on subsets $\mathcal{N}_{\lambda}^{+}$or $\mathcal{N}_{\lambda}^{-}$of $\mathcal{N}_{\lambda}$ such that $u \notin \mathcal{N}_{\lambda}^{0}$. Then $u$ is a critical point of $E_{\lambda}$.

Proof. Recall $u$ is a local minimizer for $E_{\lambda}$ under the constraint

$$
I_{\lambda}(u):=\left\langle E_{\lambda}^{\prime}(u), u\right\rangle=0 .
$$

Hence, using the theory of Lagrange multipliers, we obtain the existence of $\mu \in \mathbb{R}$ such that

Therefore,

$$
E_{\lambda}^{\prime}(u)=\mu I_{\lambda}^{\prime}(u)
$$

$$
\left\langle E_{\lambda}^{\prime}(u), u\right\rangle=\mu\left\langle I_{\lambda}^{\prime}(u), u\right\rangle=\mu \Phi_{u}^{\prime \prime}(1)=0 .
$$

So, $u \notin \mathcal{N}_{\lambda}^{0}$, hence $\Phi_{u}^{\prime \prime}(1) \neq 0$. Consenquently, $\mu=0$. The proof of Lemma 3.2 is thus complete.

Lemma 3.3. There exists $\lambda_{0}$ such that for every $0<\lambda<\lambda_{0}$, we have $\mathcal{N}_{\lambda}^{ \pm} \neq \emptyset$ and $\mathcal{N}_{\lambda}^{0}=\{0\}$.

Proof. First, by Lemma 3.2, we deduce that $\mathcal{N}_{\lambda}^{ \pm}$are nonempty for $\lambda \in\left(0, \lambda_{0}\right)$. Now, suppose that there exists $u \in \mathcal{N}_{\lambda}^{0}$ such that $\|u\|>1$. Using the definition of $\mathcal{N}_{\lambda}^{0}$, we obtain

$$
\int_{\Omega}|\nabla u|^{p(x)} d x-\int_{\Omega} a(x)|u|^{q(x)} d x-\lambda \int_{\Omega} b(x)|u|^{1-\delta(x)} d x=0 .
$$

Combining the above equality with (3.5) and Theorem 2.3 in [13], we get

$$
\begin{aligned}
0 & =\left\langle I_{\lambda}^{\prime}(u), u\right\rangle=\int_{\Omega} p(x)|\nabla u|^{p(x)} d x-\int_{\Omega} a(x) q(x)|u|^{q(x)} d x-\lambda \int_{\Omega} b(x)(1-\delta(x))|u|^{1-\delta(x)} d x \\
& \geq p^{-} \int_{\Omega}|\nabla u|^{p(x)} d x-q^{+} \int_{\Omega} a(x)|u|^{q(x)} d x-\left(1-\delta^{+}\right)\left(\int_{\Omega}|\nabla u|^{p(x)} d x-\int_{\Omega} a(x)|u|^{q(x)} d x\right) . \\
& \geq\left(p^{-}-\left(1-\delta^{+}\right)\right) \int_{\Omega}|\nabla u|^{p(x)} d x+\left(1-\delta^{+}-q^{+}\right) \int_{\Omega} a(x)|u|^{q(x)} d x .
\end{aligned}
$$

It now follows from Theorem 2.4 that

$$
\left(p^{-}-\left(1-\delta^{+}\right)\right)\|u\|^{p^{-}}+c_{10}\left(1-\delta^{+}-q^{+}\right)\|u\|^{q^{+}} \geq 0,
$$

hence

$$
\|u\| \geq c_{10}\left(\frac{p^{-}+\delta^{+}-1}{1-\delta^{+}-q^{+}}\right)^{\frac{1}{q^{+-}-p^{-}}} .
$$

In the same way, since $u \in \mathcal{N}_{\lambda}$, we obtain

$$
\int_{\Omega}|\nabla u|^{p(x)} d x-\int_{\Omega} a(x)|u|^{q(x)} d x-\lambda \int_{\Omega} b(x)|u|^{1-\delta(x)} d x=0
$$

and since $u \in \mathcal{N}_{\lambda}^{0}$, we have

$$
p^{+} \int_{\Omega}|\nabla u|^{p(x)} d x-q^{-} \int_{\Omega} a(x)|u|^{q(x)} d x-\lambda\left(1-\delta^{+}\right) \int_{\Omega} b(x)|u|^{1-\delta(x)} d x \geq 0 .
$$

Therefore

$$
\begin{aligned}
& p^{+} \int_{\Omega}|\nabla u|^{p(x)} d x-q^{-} \int_{\Omega} a(x)|u|^{q(x)} d x \\
& -\lambda\left(1-\delta^{+}\right)\left(\int_{\Omega}|\nabla u|^{p(x)} d x-\lambda \int_{\Omega} a(x)|u|^{q(x)} d x\right) \geq 0 .
\end{aligned}
$$




$$
=\left(p^{+}-q^{+}\right) \int_{\Omega}|\nabla u|^{p(x)} d x+\lambda\left(q^{+}+\delta^{+}-1\right) \int_{\Omega} b(x)|u|^{1-\delta(x)} d x \geq 0 .
$$

Now since $\|u\|>1$, by Theorem 2.4, one has

$$
\left(p^{+}-q^{+}\right)\|u\|^{p^{-}}+c_{11} \lambda\left(q^{+}+\delta^{+}-1\right)\|u\| \|^{1-\delta^{+}} \geq 0,
$$

and therefore

$$
\|u\| \leq c_{11}\left(\lambda \frac{q^{+}+\delta^{+}-1}{q^{+}-p^{-}}\right)^{\frac{1}{p^{-+\delta^{+}-1}}} .
$$

Using (3.6) and (3.7),

$$
c_{11}\left(\lambda \frac{q^{+}+\delta^{+}-1}{q^{+}-p^{-}}\right)^{\frac{1}{p^{-+\delta^{+}-1}}} \geq c_{10}\left(\frac{p^{-}+\delta^{+}-1}{1-\delta^{+}-q^{+}}\right)^{\frac{1}{q^{+}-p^{-}}} .
$$

we get

$$
\lambda \geq \frac{c_{10}}{c_{11}}\left(\frac{p^{-}+\delta^{+}-1}{1-\delta^{+}-q^{+}}\right)^{\frac{p^{-}+\delta^{+}-1}{q^{+}-p^{-}}}\left(\frac{q^{+}+\delta^{+}-1}{q^{+}-p^{-}}\right) .
$$

Then, if $\lambda$ is small enough,

$$
\lambda=\frac{c_{10}}{c_{11}}\left(\frac{p^{-}+\delta^{+}-1}{1-\delta^{+}-q^{+}}\right)^{\frac{p^{-}+\delta^{+}-1}{q^{+}-p^{-}}}\left(\frac{q^{+}+\delta^{+}-1}{q^{+}-p^{-}}\right),
$$

we obtain $\|u\|<1$, which is impossible. Therfore, $\mathcal{N}_{\lambda}^{0}=\{0\}$ for all $\lambda \in\left(0, \lambda_{0}\right)$. Hence, this completes the proof of Lemma 3.3.

\section{Existence of minimizers on $\mathcal{N}_{\lambda}^{+}$}

In this section, we shall prove the existence of a minimum for the functional energy $E_{\lambda}$ in $\mathcal{N}_{\lambda}^{+}$. We shall also prove that this minimizer is a solution to problem $\left(P_{\lambda}\right)$.

Theorem 4.1. There exists $u_{\lambda} \in \mathcal{N}_{\lambda}^{+}$satisfying

$$
E_{\lambda}\left(u_{\lambda}\right)=\inf _{u \in \mathcal{N}_{\lambda}^{+}} E_{\lambda}(u),
$$

for all $\lambda \in\left(0, \lambda_{0}\right)$.

Proof. Suppose that $\lambda \in\left(0, \lambda_{0}\right)$. Now, $E_{\lambda}$ is bounded below on $\mathcal{N}_{\lambda}$ and hence also on $\mathcal{N}_{\lambda}^{+}$. Therefore there exists a sequence $\left\{u_{n}\right\} \subset \mathcal{N}_{\lambda}^{+}$, satisfying $E_{\lambda}\left(u_{n}\right) \rightarrow$ $\inf _{u \in \mathcal{N}_{\lambda}^{+}} E_{\lambda}(u)$, as $n \rightarrow \infty$.

Since $E_{\lambda}$ is coercive, $\left\{u_{n}\right\}$ is bounded in $W_{0}^{1, p(x)}(\Omega)$. Therefore we can assume that $u_{n} \rightarrow u_{0}$ weakly in $W_{0}^{1, p(x)}(\Omega)$ and by the compact embedding, we obtain

$$
u_{n} \rightarrow u_{0} \text { in } L_{b(x)}^{1-\delta(x)}(\Omega)
$$

and

$$
u_{n} \rightarrow u_{0} \text { in } L_{a(x)}^{q(x)}(\Omega) .
$$

Now, we shall show that $u_{n} \rightarrow u_{0}$ strongly in $W_{0}^{1, p(x)}(\Omega)$. First, we shall prove that

$$
\inf _{u \in \mathcal{N}_{\lambda}^{+}} E_{\lambda}(u)<0
$$


Let $u_{0} \in \mathcal{N}_{\lambda}^{+}$. Then $\phi_{u_{0}}^{\prime \prime}(1)>0$ which gives

$p^{+} \int_{\Omega}|\nabla u|^{p(x)} d x-q^{-} \int_{\Omega} a(x)|u|^{q(x)} d x-\lambda\left(1-\delta^{+}\right) \int_{\Omega} b(x)|u|^{1-\delta(x)} d x>0$.

Moreover, by the definition of the functional energy $E_{\lambda}$, we can write

$E_{\lambda}(u) \leq \frac{1}{p^{-}} \int_{\Omega}|\nabla u|^{p(x)} d x-\frac{1}{q^{+}} \int_{\Omega} a(x)|u|^{q(x)} d x-\frac{\lambda}{1-\delta^{+}} \int_{\Omega} b(x)|u|^{1-\delta(x)} d x$.

Now, we multiply $(3.5)$ by $\left(-\left(1-\delta^{+}\right)\right)$and get

$-\left(1-\delta^{+}\right) \int_{\Omega}|\nabla u|^{p(x)} d x+\left(1-\delta^{+}\right) \int_{\Omega} a(x)|u|^{q(x)} d x+\lambda\left(1-\delta^{+}\right) \int_{\Omega} b(x)|u|^{1-\delta(x)} d x=0$.

Invoking the above equality and (4.1), one gets

$$
\int_{\Omega} a(x)|u|^{q(x)} d x<\frac{p^{+}+\delta^{+}-1}{q^{-}+\delta^{+}-1} \int_{\Omega}|\nabla u|^{p(x)} d x .
$$

On the other hand, from (3.5) and (4.2), we obtain

$E_{\lambda}(u) \leq\left(\frac{1}{p^{-}}-\frac{1}{1-\delta^{+}}\right) \int_{\Omega}|\nabla u|^{p(x)} d x-\left(\frac{1}{q^{-}}-\frac{1}{1-\delta^{+}}\right) \int_{\Omega} a(x)|u|^{q(x)} d x$.

Then, by (4.3) and (4.4), we get

$$
E_{\lambda}(u)<-\frac{\left(p^{-}+\delta^{+}-1\right)\left(q^{+}-p^{-}\right)}{p^{-} q^{+}\left(1-\delta^{+}\right)}\|u\|^{p^{-}}<0 .
$$

Now, let us assume that $u_{n} \nrightarrow u_{0}$ strongly in $W_{0}^{1, p(x)}(\Omega)$. Then

$$
\int_{\Omega}\left|\nabla u_{0}\right|^{p(x)} d x \leq \liminf _{n \rightarrow \infty} \int_{\Omega}\left|\nabla u_{n}\right|^{p(x)} d x .
$$

Using the compactness of embeddings, we obtain

$$
\begin{gathered}
\int_{\Omega} a(x) u_{0}^{q(x)} d x=\liminf _{n \rightarrow \infty} \int_{\Omega} b(x) u_{n}^{q(x)} d x, \\
\int_{\Omega} b(x) u_{0}^{1-\delta(x)} d x=\liminf _{n \rightarrow \infty} \int_{\Omega} a(x) u_{n}^{1-\delta(x)} d x .
\end{gathered}
$$

Now, by (3.5) and Theorem 2.3 in [13], one has

$$
E_{\lambda}\left(u_{n}\right) \geq\left(\frac{1}{p^{-}}-\frac{1}{q^{+}}\right) \int_{\Omega}\left|\nabla u_{n}\right|^{p(x)} d x+\lambda\left(\frac{1}{q^{+}}-\frac{1}{1-\delta^{+}}\right) \int_{\Omega} b(x)\left|u_{n}\right|^{1-\delta(x)} d x .
$$

Passing to the limit when $n$ goes to $\infty$, we obtain

$$
\begin{aligned}
& \lim _{n \rightarrow \infty} E_{\lambda}\left(u_{n}\right) \geq\left(\frac{1}{p^{-}}-\frac{1}{q^{+}}\right) \lim _{n \rightarrow \infty} \int_{\Omega}\left|\nabla u_{n}\right|^{p(x)} d x \\
& +\lambda\left(\frac{1}{q^{+}}-\frac{1}{1-\delta^{+}}\right) \lim _{n \rightarrow \infty} \int_{\Omega} b(x)\left|u_{n}\right|^{1-\delta(x)} d x .
\end{aligned}
$$

Hence, using Theorem 2.3 in [13], we get

$$
\inf _{u \in \mathcal{N}^{+}} E_{\lambda}(u)>\left(\frac{1}{p^{-}}-\frac{1}{q^{+}}\right)\left\|u_{0}\right\|^{p^{-}}+\lambda c_{5}\left(\frac{1}{q^{+}}-\frac{1}{1-\delta^{+}}\right)\left(\left\|u_{0}\right\|^{1-\delta^{-}}+\left\|u_{0}\right\|^{1-\delta^{+}}\right)>0
$$

since $p^{-}>1-\delta^{+} \geq 1-\delta^{-}$and $\left\|u_{0}\right\|>1$, which gives a contradiction. Therefore, $u_{n} \rightarrow u_{0}$ strongly in $W_{0}^{1, p(x)}(\Omega)$ and $E_{\lambda}\left(u_{0}\right)=\inf _{u \in \mathcal{N}_{\lambda}^{+}} E_{\lambda}(u)$. This completes the proof of Theorem 4.1. 


\section{EXISTENCE OF Minimizers ON $\mathcal{N}_{\lambda}^{-}$}

In this section, we shall prove the existence of a minimum for the functional energy $E_{\lambda}$ in $\mathcal{N}_{\lambda}^{-}$. We shall also prove that this minimizer is a solution to problem $\left(P_{\lambda}\right)$.

Theorem 5.1. There exists $v_{\lambda} \in \mathcal{N}_{\lambda}^{-}$such that

$$
E_{\lambda}\left(v_{\lambda}\right)=\inf _{v \in \mathcal{N}_{\lambda}^{-}} E_{\lambda}(v),
$$

for all $\lambda \in\left(0, \lambda_{0}\right)$.

Proof. Suppose that $\lambda \in\left(0, \lambda_{0}\right)$. Since $E_{\lambda}$ is bounded below on $\mathcal{N}_{\lambda}$ hence also on $\mathcal{N}_{\lambda}^{-}$. Therefore, there exists a sequence $\left\{v_{n}\right\} \subset \mathcal{N}_{\lambda}^{-}$, satisfying $E_{\lambda}\left(v_{n}\right) \rightarrow$ $\inf _{u \in \mathcal{N}_{\lambda}^{-}} E_{\lambda}(u)$, as $n \rightarrow \infty$. Since $E_{\lambda}$ is coercive, $\left\{v_{n}\right\}$ is bounded in $W_{0}^{1, p(x)}(\Omega)$.

Therefore we can assume that $v_{n} \rightarrow v_{0}$ weakly in $W_{0}^{1, p(x)}(\Omega)$ and by the compact embedding, we get

$$
v_{n} \rightarrow v_{0} \text { in } L_{b(x)}^{1-\delta(x)}(\Omega)
$$

and

$$
v_{n} \rightarrow v_{0} \text { in } L_{a(x)}^{q(x)}(\Omega) .
$$

Now, we shall show $v_{n} \rightarrow v_{0}$ strongly in $W_{0}^{1, p(x)}(\Omega)$. First, we shall prove that

$$
\inf _{v \in \mathcal{N}_{\lambda}^{-}} E_{\lambda}(v)>0
$$

Let $v_{0} \in \mathcal{N}_{\lambda}^{-}$. Then we have from (3.5),

$$
\int_{\Omega}|\nabla u|^{p(x)} d x-\int_{\Omega} a(x)|u|^{q(x)} d x-\lambda \int_{\Omega} b(x)|u|^{1-\delta(x)} d x=0 .
$$

Moreover, by the definition of the functional energy $E_{\lambda}$, we can write

$$
E_{\lambda}(v) \geq \frac{1}{p^{-}} \int_{\Omega}|\nabla v|^{p(x)} d x-\frac{1}{q^{+}} \int_{\Omega} a(x)|v|^{q(x)} d x-\frac{\lambda}{1-\delta^{+}} \int_{\Omega} b(x)|v|^{1-\delta(x)} d x .
$$

Hence, from (5.1) and (5.2), one has

$$
\begin{aligned}
& E_{\lambda}(v) \geq \frac{1}{p^{-}} \int_{\Omega}|\nabla v|^{p(x)} d x-\frac{\lambda}{1-\delta^{+}} \int_{\Omega} b(x)|v|^{1-\delta(x)} d x \\
& -\frac{1}{q^{+}}\left(\int_{\Omega}|\nabla v|^{p(x)} d x-\lambda \int_{\Omega} b(x)|v|^{1-\delta(x)} d x\right) \\
& \geq\left(\frac{1}{p^{-}}-\frac{1}{q^{+}}\right) \int_{\Omega}|\nabla v|^{p(x)} d x+\left(\frac{1}{q^{+}}-\frac{1}{1-\delta^{+}}\right) \int_{\Omega} b(x)|v|^{1-\delta(x)} d x \\
& \geq\left(\frac{1}{p^{-}}-\frac{1}{q^{+}}\right)\|v\|^{p^{-}}+\lambda c_{8}\left(\frac{1}{q^{+}}-\frac{1}{1-\delta^{+}}\right)\|v\|^{1-\delta^{+}} \\
& \geq\left[\left(\frac{1}{p^{-}}-\frac{1}{q^{+}}\right)+\lambda c_{8}\left(\frac{1}{q^{+}}-\frac{1}{1-\delta^{+}}\right)\right]\|v\|^{p-}
\end{aligned}
$$

since $p^{-}>1-\delta^{+}$.

Hence, if we choose

$$
\lambda<\frac{\left(1-\delta^{+}\right)\left(p^{-}-q^{+}\right)}{c_{8} p^{+}\left(1-\delta^{+}-q^{+}\right)},
$$


we obtain $E_{\lambda}(v)>0$. Moroever, since $\mathcal{N}_{\lambda}^{+} \cap \mathcal{N}_{\lambda}^{-}=\emptyset$ and $\inf _{v \in \mathcal{N}_{\lambda}^{+}} E_{\lambda}(v)<0$, we see that $v \in \mathcal{N}_{\lambda}^{-}$.

In the same way, if $v_{0} \in \mathcal{N}_{\lambda}^{-}$, hence there exists $t_{0}$ satisfying $t_{0} v_{0} \in \mathcal{N}_{\lambda}^{-}$and so $E_{\lambda}\left(t_{0} v_{0}\right) \leq E_{\lambda}\left(v_{0}\right)$. Moreover, since

$I_{\lambda}^{\prime}(v)=\int_{\Omega} p(x)|\nabla v|^{p(x)} d x-\int_{\Omega} a(x) q(x)|v|^{q(x)} d x-\lambda \int_{\Omega} b(x)(1-\delta(x))|v|^{1-\delta(x)} d x$,

we get

$$
\begin{aligned}
& I_{\lambda}^{\prime}\left(t_{0} v_{0}\right)=\int_{\Omega} p(x)\left|\nabla t_{0} v_{0}\right|^{p(x)} d x-\int_{\Omega} a(x) q(x)\left|t_{0} v_{0}\right|^{q(x)} d x-\lambda \int_{\Omega} b(x)(1-\delta(x))\left|t_{0} v_{0}\right|^{1-\delta(x)} d x \\
& \leq t_{0}^{p^{+}} p^{+} \int_{\Omega}\left|\nabla v_{0}\right|^{p(x)} d x-t_{0}^{q^{-}} q^{-} \int_{\Omega} a(x)\left|v_{0}\right|^{q(x)} d x-\lambda\left(1-\delta^{+}\right) t_{0}^{1-\delta^{+}} \int_{\Omega} b(x)\left|v_{0}\right|^{1-\delta(x)} d x,
\end{aligned}
$$

since $1-\delta^{+}<p^{+}<q^{-}$. By the conditions on $a$ and $b$ it follows that $I_{\lambda}^{\prime}\left(t_{0} v_{0}\right)<0$, so by definition of $\mathcal{N}_{\lambda}^{-} t_{0} v_{0} \in \mathcal{N}_{\lambda}^{-}$.

Now, let us assume that $v_{n} \nrightarrow v_{0}$ strongly in $W_{0}^{1, p(x)}(\Omega)$. Using the fact that

$$
\int_{\Omega}\left|\nabla v_{0}\right|^{p(x)} d x \leq \liminf _{n \rightarrow \infty} \int_{\Omega},\left|\nabla v_{n}\right|^{p(x)} d x
$$

one gets

$$
\begin{aligned}
& E_{\lambda}\left(t v_{0}\right) \leq \int_{\Omega} \frac{t^{p(x)}\left|\nabla v_{0}\right|^{p(x)}}{p(x)} \mathrm{d} x-\int_{\Omega} \frac{t^{q(x)}}{q(x)}\left|v_{0}\right|^{q(x)} d x-\lambda \int_{\Omega} \frac{t^{1-\delta(x)}\left|v_{0}\right|^{1-\delta(x)}}{1-\delta(x)} d x, \\
& \leq \lim _{n \rightarrow \infty}\left[\frac{t^{p^{+}}}{p^{-}} \int_{\Omega}\left|\nabla v_{n}\right|^{p(x)} \mathrm{d} x-\frac{t^{q^{-}}}{q^{+}} \int_{\Omega}\left|v_{n}\right|^{q(x)} d x-\lambda \frac{t^{1-\delta^{+}}}{1-\delta^{+}} \int_{\Omega}\left|v_{n}\right|^{1-\delta(x)} d x\right], \\
& \leq \lim _{n \rightarrow \infty} E_{\lambda}\left(t v_{n}\right) \leq \lim _{n \rightarrow \infty} E_{\lambda}\left(v_{n}\right)=\inf _{v \in \mathcal{N}_{\lambda}^{-}} E_{\lambda}(v),
\end{aligned}
$$

which contradicts with the fact that $t v_{0} \in \mathcal{N}_{\lambda}^{-}$. Hence, $v_{n} \rightarrow v_{0}$ strongly in $W_{0}^{1, p(x)}(\Omega)$ and $E_{\lambda}\left(v_{0}\right)=\inf _{v \in \mathcal{N}_{\lambda}^{-}} E_{\lambda}(v)$. This completes the proof of Theorem 5.1 .

\section{Proof of Theorem 1.1}

Proof. By Theorem 4.1 and Theorem 5.1, for all $\lambda \in\left(0, \lambda_{0}\right)$, there exist $u_{0} \in \mathcal{N}_{\lambda}^{+}$, $v_{0} \in \mathcal{N}_{\lambda}^{-}$such that

$$
E_{\lambda}\left(u_{0}\right)=\inf _{u \in \mathcal{N}_{\lambda}^{+}} E_{\lambda}(u)
$$

and

$$
E_{\lambda}\left(v_{0}\right)=\inf _{v \in \mathcal{N}_{\lambda}^{-}} E_{\lambda}(v)
$$

On the other hand, since $E_{\lambda}\left(u_{0}\right)=E_{\lambda}\left(\left|u_{0}\right|\right)$ and $\left|u_{0}\right| \in \mathcal{N}_{\lambda}^{+}$and in the same way, $E_{\lambda}\left(v_{0}\right)=E_{\lambda}\left(\left|v_{0}\right|\right)$ and $\left|v_{0}\right| \in \mathcal{N}_{\lambda}^{-}$, we suppose that $u_{0}, v_{0} \geq 0$. Using Lemma $3.2, u_{0}, v_{0}$ are critical points of $E_{\lambda}$ on $W_{0}^{1, p(x)}(\Omega)$ and thus weak solutions of $\left(\mathrm{P}_{\lambda}\right)$.

Finally, by the Harnack inequality and by ZHANG-LiU [26], we obtain that $u_{0}, v_{0}$ are nonnegative solutions of $\left(\mathrm{P}_{\lambda}\right)$.

It remains to prove that the solutions we obtained for Theorem 4.1 and Theorem 5.1 are distinct. Indeed, since $\mathcal{N}_{\lambda}^{-} \cap \mathcal{N}_{\lambda}^{+}=\emptyset$, it follows that $u_{0}$ and $v_{0}$ are different. This completes the proof of Theorem 1.1. 


\section{ACKNOWLEDGEMENTS}

The first author was supported by the Slovenian Research Agency program P10292 and grants N1-0114 and N1-0083.

\section{REFERENCES}

[1] E. Acerbi And G. Mingione, Regularity results for a class of functionals with nonstandard growth, Arch. Rational Mech. Anal. 156 (2001) 121-140.

[2] M.M. Coclite and G. Palmieri, On a singular nonlinear Dirichlet problem, Comm. Partial Differential Equations 14 (1989), 1315-1327.

[3] M.G. Crandall, P.H. Rabinowitz and L. Tartar, On a Dirichlet problem with a singular nonlinearity, Comm. Partial Differential Equations 2 (1977), 193-222.

[4] L. Diening, Theorical and numerical results for electrorheological fluids, Ph.D. Thesis, University of Freiburg, Freiburg, 2002.

[5] P. Drabek and S.I. Pohozaev, Positive solutions for the p-Laplacian: application of the fibering method, Proc. Royal Soc. Edinburgh Sect A, 127 (1997), 703-726.

[6] X. L. FAN, Solutions for $p(x)$-Laplacian Dirichlet problems with singular coefficients, J. Math. Anal. Appl. 312 (2005), 464-477.

[7] X.L. Fan, J.S. Shen and D. ZhaO Sobolev embedding Theorems for spaces $W^{k, p(x)}(\Omega)$, J. Math. Anal. Appl. 262 (2001), 749-760.

[8] X.L. Fan And D. ZhaO, On spaces $L^{p(x)}(\Omega)$ and $W^{m, p(x)}(\Omega)$, J. Math. Anal. Appl. bf 263 (2001), 424-446.

[9] M. Ghergu And V. RĂdulescu, Singular Elliptic Problems: Bifurcation and Asymptotic Analysis, Oxford Lecture Series in Mathematics and its Applications 37. Oxford University Press, Oxford, 2008.

[10] J. Giacomoni And K. SaOudi, Multiplicity of positive solutions for a singular and critical problem, Nonlinear Anal. 71 (2009), no. 9, 4060-4077.

[11] J. Giacomoni, I. Schindler And P. TAKÁC̆, Sobolev versus Hölder local minimizers and global multiplicity for a singular and quasilinear equation, Annali della Scuola Normale Superiore di Pisa, Classe di scienze Série V 6 (2007), 117-158.

[12] O. KovăČIK And J. RĂKosnik, On spaces $L^{p(x)}$ and $W^{k, p(x)}$, Czechoslovak Math. J. 41 (116) (1991), 592-618.

[13] R.A. Mashiyev, S. Ogras, Z. Yucedag and M. Avci, The Nehari manifold approach for Dirichlet problem involving the $p(x)$-Laplacian equation, J. Korean Math. Soc. 47 (2010), No. 1. pp. 1-16.

[14] N.S. Papageorgiou, V.D. Rădulescu, D.D. Repovš, Nonlinear Analysis - Theory and Methods, Springer Monographs in Mathematics, Springer, Cham, 2019.

[15] V.D. Rădulescu and D.D. Repovš, Partial Differential Equations with Variable Exponents: Variational Methods and Qualitative Analysis, Chapman and Hall /CRC, Taylor \& Francis Group, Boca Raton, FL, 2015.

[16] S. Saiedinezhad And M.B. Ghaemi, The fibering map approach to a quasilinear degenerate $p(x)$-Laplacian equation, Bull. Iranian Math. Soc., 41 (2015), 1477-1492.

[17] K. SAOUdI, Existence and non-existence of solution for a singular nonlinear Dirichlet problem involving the $p(x)$-Laplace operator, J. Adv. Math. Stud. 9 (2016), No. 2, 292-303.

[18] K. SAOUdI, Existence and non-existence for a singular problem with variables potentials, Electronic Journal of Differential equations 2017 (2017), No. 291, 1-9.

[19] SAOUdI, A singular elliptic system involving the $p(x)$-Laplacian and generalized Lebesgue-Sobolev spaces, International Journal of Mathematics 30(116):1950064 (2019).

[20] K. SaOudi And A. Ghanmi, A multiplicity results for a singular problem involving the fractional p-Laplacian operator, Complex Var. Elliptic Equ. 61:9 (2016), 1199-1216. 
[21] K. Saoudi And A. Ghanmi, A multiplicity results for a singular equation involving the $p(x)$-Laplace operator, Complex Var. Elliptic Equ. 62 (2017) No. 5, 695-725.

[22] K. SAOUdi AND M. Kratou, Existence of multiple solutions for a singular and quasilinear equation, Complex Var. Elliptic Equ. 60 (2015), no.7, 893-925.

[23] K. Saoudi, M. Kratou and S. Al Sadhan, Multiplicity results for the $p(x)$ Laplacian equation with singular nonlinearities and nonlinear Neumann boundary condition, Intern. J. Differential Equ. 2016 (2016), Article ID 3149482, 14 pp.

[24] Q.H. ZHANG, A strong maximum principle for differential equations with nonstandard $p(x)$-growth conditions, J. Math. Anal. Appl. 312 (2005), No.1, 24-32.

[25] Q. ZHANG, Existence and asymptotic behavior of positive solutions to $p(x)$-Laplacian equations with singular nonlinearities, J. Inequal. Appl. 2007, Art. ID 19349, 9 pp.

[26] X. Zhang And X. Liu, The local boundedness and Harnack inequality of $p(x)$ - Laplace equation, J. Math. Anal. Appl. 332 (2007), 209-218. 